\title{
Mapa de la estructura intelectual de El profesional de la información de 2014 a 2018
}

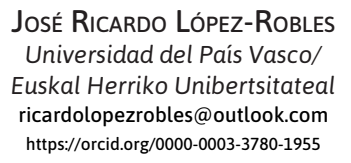

JAVIER GUALLAR

Universitat de Barcelona jguallar@ub.edu

https://orcid.org/0000-0002-8601-3990

\author{
Nadia Karina Gamboa-Rosales \\ Universidad Autónoma \\ de Zacatecas, México \\ ngamboa@conacyt.mx \\ https://orcid.org/0000-0003-3421-8289
}

\author{
Jose Ramón Otegi-Olaso \\ Universidad del País Vasco/ \\ Euskal Herriko Unibertsitateal \\ joserra.otegi@ehu.eus
} https://orcid.org/0000-0001-6023-8537
Manuel Jesús Cobo

Universidad de Cádiz

manueljesus.cobo@uca.es

http://orcid.org/0000-0001-6575-803X

\section{Mapping the intellectual structure of El profesional de la información from 2014 to 2018}

\section{RESUMEN ABSTRACT}

El profesional de la información (EPI) es una de las principales revistas científicas españolas sobre información, comunicación, bibliotecas y nuevas tecnologías de la información incluida en índices internacionales como los JCR, y SJR. EPI cuenta con una trayectoria de más de 20 años, lo que le ha convertido en una fuente de referencia y escaparate para el desarrollo de investigaciones en los ámbitos antes mencionados. En este sentido, resulta necesario entender cuáles son las principales líneas que se están desarrollando dentro del marco de la revista, sus principales autores y el impacto que tienen sus publicaciones en el desarrollo del estado del arte. En respuesta a esta necesidad, esta publicación analiza los últimos cinco años (2014-2018) de EPI utilizando SciMAT como herramienta bibliométrica para la creación de mapas de ciencia, identificando las áreas temáticas que han sido objeto de investigación durante el periodo de análisis, su composición, relación y peso en la evolución de la revista.
El profesional de la información (EPI) is one of the main Spanish scientific journals on information, communication, indicators, libraries and new information technologies included in international indexes such as JCR and SJR. EPI has a track record of more than twenty years, and it has become a referent source and good place to disseminate the advances y results of research and projects within the knowledge areas mentioned above. In this respect, it is necessary to understand the main themes that are being developed within the journal's framework, its main authors, organizations and countries, and the impact that its publications have on the development of the state of the art. In response to this need, this publication analyses the last five years (2014-2018) of EPI using SciMAT as a bibliometric tool for the creation of science maps, identifying the thematic areas that have been the object of research during the period of analysis, their composition, relationship and relevance in the evolution of the journal.
PALABRAS CLAVE

Revistas científicas; Bibliometría; Estructura conceptual; SciMAT; El profesional de la información; Información y Documentación; Comunicación; Inteligencia Estratégica

\section{KEYWORDS}

Academic journals, Bibliometrics; Intellectual structure; SciMAT; El profesional de la información; Library and Information Science; Communication; Strategic Intelligence 


\section{Introducción}

Hoy en día las revistas científicas se han convertido en uno de los principales medios de difusión de avances, resultados y proyectos, tanto para académicos y científicos como para profesionales. Esta situación, a su vez, está permitiendo utilizar estos recursos como fuente de información para otros fines, ya que a través de las revistas es posible conocer quiénes son los autores u organizaciones con una mayor productividad e impacto, así como las líneas de investigación y especialización que estos están siguiendo para mejorar su posicionamiento y el estado del arte, entre otros aspectos (Abadal, 2017; López-Ornelas y Cordero-Arroyo, 2005).

Sin embargo, no existe un único procedimiento para poder analizar este tipo de recursos. Atendiendo a esta oportunidad, desde la bibliometría, entendida como la ciencia de análisis y evaluación de los campos científicos (Callon, Courtial y Penan, 1995; Castillo-Esparcia, Rubio-Moraga y Almansa-Martínez, 2012), se está trabajando en la definición de indicadores, metodologías, herramientas y técnicas que permitan estudiar el rendimiento y evolución de un concepto, un área de conocimiento o inclusive un agente.

En este sentido, en el caso de las revistas científicas es posible resaltar tres tipos de investigaciones que las tienen por objeto de estudio: (i) Estudios bibliométricos de rendimiento sobre producción y autoría, que se centran en analizar los perfiles de los autores, atendiendo a elementos como su país, filiación o género, y la producción de artículos, examinando cuáles son los más relevantes o citados, (ii) Estudios bibliométricos sobre temáticas: se centran en abordar cuáles son las principales temáticas desarrolladas, así como su evolución y relaciones, y (iii) Estudios sobre metodologías de investigación, que se centran en cuáles son las técnicas y métodos de investigación con los que se han construido los artículos publicados en las revistas (López-Robles, Guallar, Otegi-Olaso y Gamboa-Rosales, 2019).

A manera de ejemplo, es posible identificar trabajos que se centran en uno de estos enfoques (Calderon Garrido y Carnicer, 2018; López-Robles, Otegi-Olaso, Porto-Gómez y Cobo, 2019); en dos de ellos (Guallar, Ferran Ferrer, Abadal y Server, 2017; López-Robles, Otegi-Olaso, Gamboa-Rosales, Gamboa-Rosales y Cobo, 2018; López-Robles, Otegi-Olaso, Porto-Gómez, et al., 2018; López-Robles, Otegi-Olaso y Porto Gómez, 2018); o en los tres (Ferran-Ferrer, Guallar, Abadal y Server, 2017; López-Robles, Otegi-Olaso, Porto-Gómez, Gamboa-Rosales y Gamboa-Rosales, 2019; López-Robles, Otegi-Olaso, Arcos, Gamboa-Rosales y Gamboa-Rosales, 2018; López-Robles, Otegi-Olaso, Porto Gómez y Cobo, 2019).

Desde otro punto de vista también podemos distinguir entre enfoques orientados a varias revistas o a una sola publicación: (i) estudios que analizan varias revistas en conjunto o un grupo significativo de las mismas (Delgado-López-Cózar, 2001; Goyanes, Rodríguez-Gómez y Rosique-Cedillo, 2018); Somoza, Guallar, Rodríguez-Gairín y Abadal (2017), y (ii) investigaciones que se centran en analizar en profundidad una sola publicación, habitualmente tratando su evolución a lo largo de un período determinado de tiempo (Abadal, 2018; Arquero Avilés y Río Sadornil, 2002; Gonzalez-Alcaide, Gorraiz y Hervas-Oliver, 2018; López López, Díaz Hernández, Angulo Benítez y Giménez Toledo, 2001; Pérez Álvarez-Ossorio, 1997).

Tomando en cuenta lo anterior, y partiendo de los resultados preliminares expuestos en la Conferencia Internacional sobre revistas científicas CRECS2019 sobre la estructura intelectual de la revista EPI durante el último lustro (López-Robles, Cobo, Guallar, Otegi-Olaso y Gamboa-Rosales, 2019), se plantea el siguiente análisis profundizando en el rendimiento de las redes temáticas y su composición. En este sentido, las redes temáticas permiten visualizar la relación que existe entre los principales componentes de los temas identificados en el diagrama estratégico para el periodo 2014-2018. Además, de conocer la relación que existe entre los principales componentes, es posible visualizar la intensidad de esta relación, favoreciendo con ello el análisis de los elementos que promueven el desarrollo de un tema en concreto.

La revista EPI es una de las principales revistas científicas españolas sobre información, comunicación, bibliotecas y nuevas tecnologías de la información incluida en índices internacionales como los JCR, y SJR. Además, cuenta con una trayectoria de más de 20 años, lo que le ha convertido en una fuente de referencia y escaparate para el desarrollo de investigaciones en los ámbitos antes mencionados. Con anterioridad, se ha realizado un estudio sobre la revista; en este caso se abarca un período de tiempo diferente, centrándonos en los cinco años más recientes, 2014-2018 (López-Robles, Guallar, et al., 2019).

\section{Metodología y preparación de datos}

\subsection{Metodología}

Para establecer la estructura intelectual de la revista EPI e identificar las principales áreas temáticas que cubre en el periodo 2014-2018, así como sus componentes, se plantea un enfoque bibliométrico basado en el desarrollo del mapa estratégico y sus mapas de redes utilizando el software SciMAT. En este caso, los mapas tienen por objetivo representar de forma espacial cómo las publicaciones a través de las palabras clave se encuentran interrelacionadas. Este método, es utilizado ampliamente también para entender, descubrir y mostrar relaciones ocultas o difíciles de ver entre temas de interés para el desarrollo de un área o campo de conocimiento.

Para ello, se ha seguido una metodología estructurada en cuatro etapas utilizando SciMAT. Esta metodología favorece 


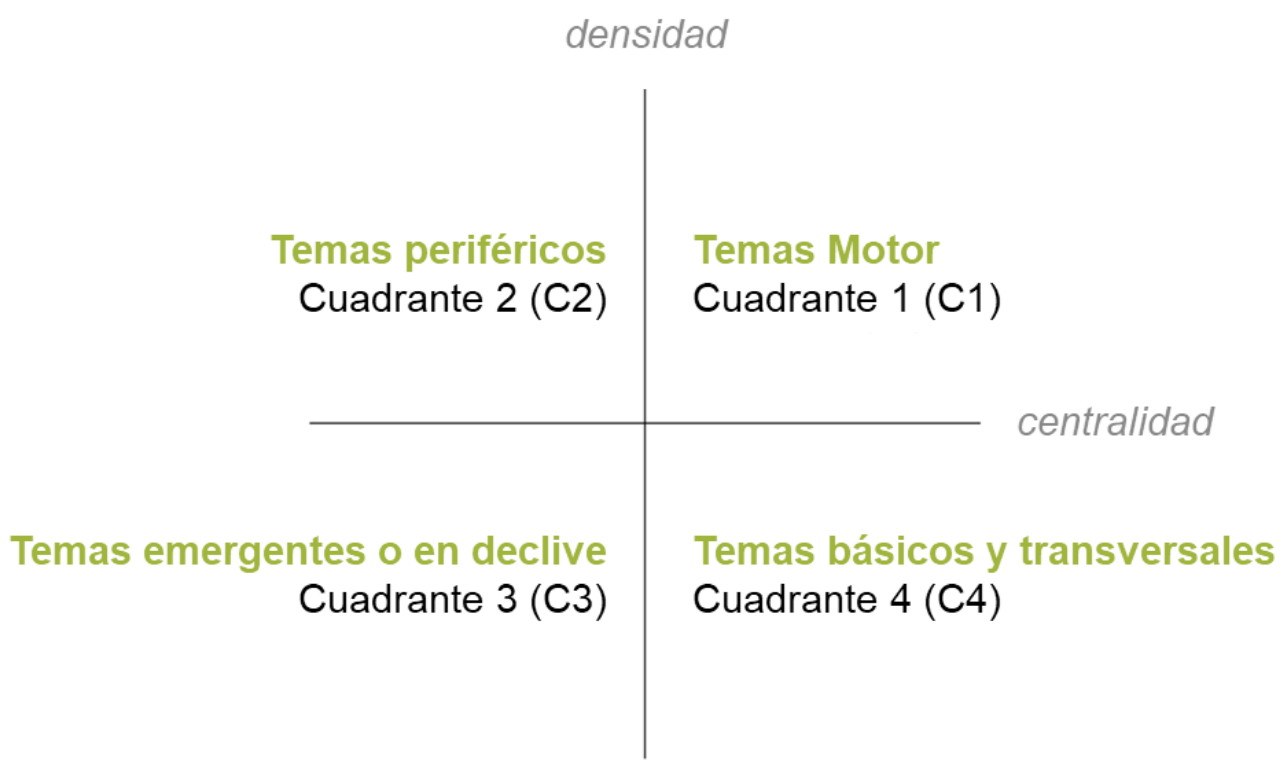

Figura 1. Diagrama estratégico según metodología SciMAT. Elaboración propia.

el desarrollo de una visión completa de la revista e individual de los propios temas (Cobo, López-Herrera, Herrera-Viedma y Herrera, 2012):

- Identificación de temas de investigación (Powell, Kouropalatis, Morgan y Karhu, 2016),

- Visualización de los temas de investigación y sus redes temáticas (Callon, Courtial y Laville, 1991; He, 1999),

- Descubrimiento de áreas temáticas (Sternitzke y Bergmann, 2009)

- Análisis de rendimiento (Cobo et al., 2012).

A través de los mapas resultantes de la metodología, es posible visualizar de forma clara la evolución de la revista, delimitando las áreas de investigación y el tiempo, capturando su estructura conceptual y cognitiva (Cobo, López-Herrera, Herrera-Viedma y Herrera, 2011). Además, a través del mapa estratégico, se caracteriza el periodo analizado a manera de red temática y se representa como un conjunto de temas clasificados y posicionados en un plano dividido en cuatro categorías según los indicadores de centralidad y densidad de Callon. La centralidad mide el grado de interacción de una red con respecto a otras redes en base a las palabras clave, reflejando con ello el grado de fuerza de los enlaces externos entre temas. Esta medida se puede interpretar como la importancia de un tema en el desarrollo del campo de conocimiento analizado o el grado de cohesión que existe con otros temas. Por su parte, la densidad, mide la fuerza interna de una red atendiendo a los enlaces que describen un tema. Tomando en cuenta ambos indicadores es posible valorar la robustez del área evaluada (ver figura 1):

- Temas motor (Cuadrante (1): Temas desarrollados e importantes para la construcción del campo científico.

- Temas periféricos (Cuadrante C2): Temas desarrollados internamente pero que se encuentran aislados del resto de temas, y que tienen un papel marginal en el desarrollo del campo científico.

- Temas emergentes o en declive (Cuadrante C3): Temas pocos desarrollados.

- Temas básicos o transversales (Cuadrante (4): Temas importantes para el desarrollo del campo científico pero que cuentan con poco desarrollo.

Estas redes temáticas se construyen según la frecuencia de co-aparición de palabras clave y se agrupan utilizando el algoritmo de centros simples. Este algoritmo refleja agrupaciones con etiquetas del nodo más central; es decir, del grupo de palabras claves aquella con mayor peso es la que agrupa a sus relaciones con menor peso. Una vez construida la red bibliométrica de co-palabras, se calcula la similitud a partir de la co-ocurrencia de las palabras clave identificadas en el conjunto de publicaciones.

\subsection{Preparación de datos}

Para el desarrollo del análisis, se han descargado las publicaciones de El profesional de la información (EPI) y su información bibliográfica de la Web of Science. Para ello, se definió una consulta avanzada utilizando el ISBN de la revista (1386-6710), y posteriormente se limitaron los resultados a 


\section{Publicaciones $\longrightarrow$ Citas de las publicaciones}

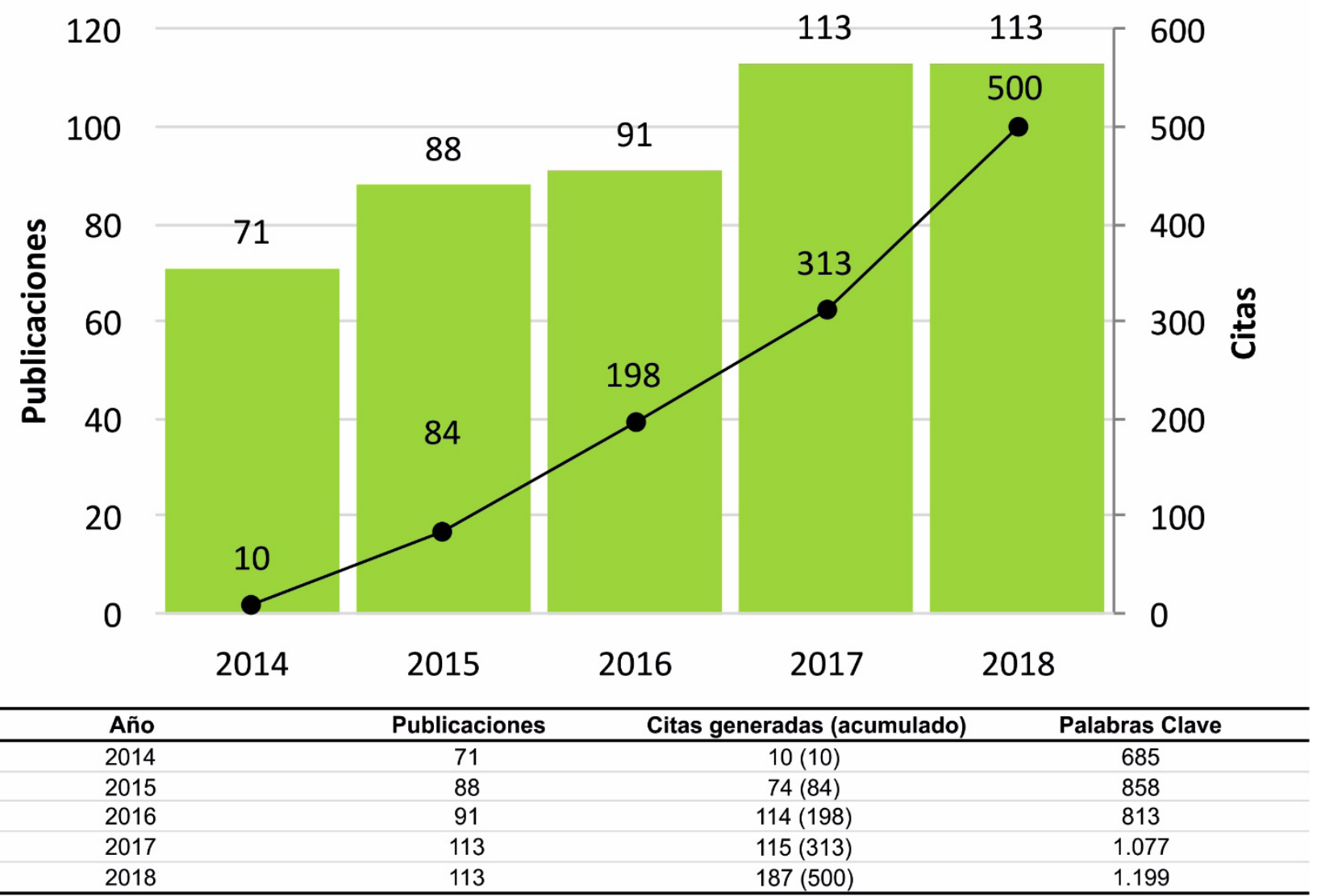

Figura 2. Evolución productiva de las publicaciones de EPI comprendidas entre 2014 y 2018. Elaboración propia.

la disponibilidad de artículos (articles) y revisiones (review) entre 2014 y 2018. En este sentido, la consulta utilizada fue: IS=("1386-6710") AND PY=2014-2018 (ARTICLE OR REVIEW).

Esta consulta recuperó un total de 476 publicaciones, las cuales concentran un total de 1.105 citas y 2.435 palabas clave al 10 de mayo de 2019, tal como se puede observar en la figura 2.

Las publicaciones identificadas en Web of Science Core Collection fueron descargadas en formato tipo texto plano y se incluyeron en el software de bibliometría SciMAT para confeccionar la base de conocimiento que permite realizar el análisis del mapa bibliométrico o científico. Asimismo, los datos bibliográficos disponibles en Web of Science (p. ej. afiliaciones, autores, referencias, fecha de publicación, palabras claves y citas obtenidas, principalmente) disponibles para cada publicación también fueron incluidos. Estos datos, permiten analizar la composición, identificar relaciones y, en consecuencia, a obtener mejores resultados en el análisis. Finalmente, para mejorar y asegurar la calidad de los datos, se aplicó un proceso de revisión individual, en el cual se repasó y agrupo uno a uno aquellos conceptos que representan un mismo tema o tienen el mismo significado, por ejemplo: "INFORMA-
TION MANAGEMENT", "INFORMATION-MANAGEMENT" y "IM" se agruparon como "INFORMATION-MANAGEMENT-(IM)".

\section{Resumen bibliométrico y conceptual de EPI entre 2014-2018}

\subsection{Resumen bibliométrico}

El profesional de la información (EPI) es una de las principales revistas españolas sobre comunicación, información, indicadores, bibliotecas y tecnologías de la información, y se encuentra indexada en las principales bases de datos. Desde 2006 a la fecha, EPI cuenta con más de 1.200 publicaciones, de las cuales más de 975 son de carácter abierto, y concentra más de 4.000 citas en más de 2.668 artículos. Estos indicadores, permiten visualizar el alcance de la revista, siendo el objeto de estudio de la presente investigación.

A continuación se presentan los autores más productivos, organizaciones más productivas y países más productivos entre 2014 y 2018. 


\begin{tabular}{|c|c|}
\hline Rendimiento & Resultados \\
\hline $\begin{array}{l}\text { Autores más } \\
\text { productivos }\end{array}$ & $\begin{array}{l}\text { (8) Pérez-Montoro } \\
\text { (7) Codina; Thelwall; Torres-Salinas } \\
\text { (6) Goyanes } \\
\text { (5) Campos-Freire; Guallar; Martí- } \\
\text { nez-Fernández; Robinson-García } \\
\text { (4) Alberich-Pascual; Arci- } \\
\text { la-Calderón; Bornmann; } \\
\text { Casero-Ripolles; Delgado-López-Co- } \\
\text { zar; Ferrán-Ferrer; Guerrero-Sole; } \\
\text { Iglesias-García; Jiménez-Contreras; } \\
\text { López-García; Manfredi-Sánchez; Mico- } \\
\text { Sanz; Orduña-Malea; Rodríguez-Bravo; } \\
\text { Rodríguez-Gairin; Rodríguez-Vázquez }\end{array}$ \\
\hline $\begin{array}{l}\text { Organizaciones } \\
\text { más productivas }\end{array}$ & $\begin{array}{l}\text { (34) Universitat Pompeu Fabra } \\
\text { (32) Universidad Complutense de } \\
\text { Madrid; Universidad Carlos III se Madrid } \\
\text { (27) Universitat de Barcelona; } \\
\text { Universidad de Granada } \\
\text { (24) Universidad Rey Juan Carlos } \\
\text { (21) Universitat Oberta de Catalunya }\end{array}$ \\
\hline $\begin{array}{l}\text { Países más } \\
\text { productivos }\end{array}$ & $\begin{array}{l}\text { (428) España } \\
\text { (14) Inglaterra } \\
\text { (10) Colombia; México } \\
\text { (7) Estados Unidos } \\
\text { (6) Chile; Alemania }\end{array}$ \\
\hline
\end{tabular}

Tabla 1. Autores, organizaciones y países más productivos en la revista EPI entre 2014 y 2018. Elaboración propia.

Tomando en cuenta el rendimiento global de la revista EPI y durante el periodo analizado, es posible visualizar la evolución y aceptación que tiene dentro de la comunidad académica, científica y profesional. Además, se puede inferir que mantendrá un crecimiento para los próximos años.

Otro aspecto a resaltar, es la internacionalización de la revista, ya que cuenta con una importante participación de investigadores de fuera de España, aspecto que puede favorecer la diseminación de la misma, por el efecto de difusión que pueden realizar estos investigadores dentro de sus redes de contactos. Además, motiva la necesidad de conocer su estructura intelectual, para identificar qué aspectos son los que le hacen atractiva para organizaciones que se encuentran fuera del alcance local y regional. En el siguiente apartado se pre- senta el análisis conceptual de EPI.

\subsection{Análisis conceptual de EPI entre 2014 y 2018}

Por otro lado, el análisis conceptual a través del uso de mapas bibliométricos o científicos permite evaluar dentro de un marco temporal la estructura conceptual, social e intelectual de un concepto, área de conocimiento o agente, a partir de su rendimiento bibliométrico y la información bibliográfica existente (Cobo, 2012). En este caso se ha evaluado la evolución de la revista EPI identificando y visualizando los principales temas de investigación desde 2014 hasta 2018 utilizando SciMAT.

Para visualizar y analizar la estructura intelectual de EPI, se ha desarrollado el mapa estratégico de los temas principales entre 2014 y 2018, así como los mapas conceptuales para cada uno de los temas identificados. El mapa estratégico cubre un periodo de los últimos cinco años completos de la revista y va acompañado de información complementaria como número de publicaciones, citas, promedio de citas, rol dentro de la estructura intelectual y su índice-h. Además, estos mapas permiten clasificar los principales temas de investigación en cuatro categorías, según su relevancia: Temas motores (Cuadrante Q1, superior derecho), Temas periféricos (Cuadrante Q2, superior izquierdo), Temas emergentes o en declive (Cuadrante Q3, inferior izquierdo) y Temas básicos y transversales (Cuadrante Q4, inferior derecho).

En la figura 3 se muestra el diagrama estratégico para el periodo entre 2014 y 2018, incluyendo el número de publicaciones y citas entre paréntesis para cada una de las líneas según los datos obtenidos de la consulta avanzada en la Web of Science Core Collection. Es importante tener presente que una misma publicación puede estar incluida en varios temas, en función de las palabras utilizadas para su clasificación, y que los Temas motores y Temas básicos y transversales son considerados como los temas que favorecen el desarrollo y consolidación de un campo de conocimiento o una revista por su densidad y centralidad.

Por otro lado, en la figura 4 se muestran los mapas de red para cada uno de los temas identificados durante el periodo 2014 2018, incluyendo los temas cubiertos por estas, su relación directa con el tema central, así como las interacciones entre ellas. Es importante indicar, que el grosor de la línea que une a las temáticas corresponde al número de documentos que comparten, así como el tamaño de la esfera al número de publicaciones que concentra cada tema. 


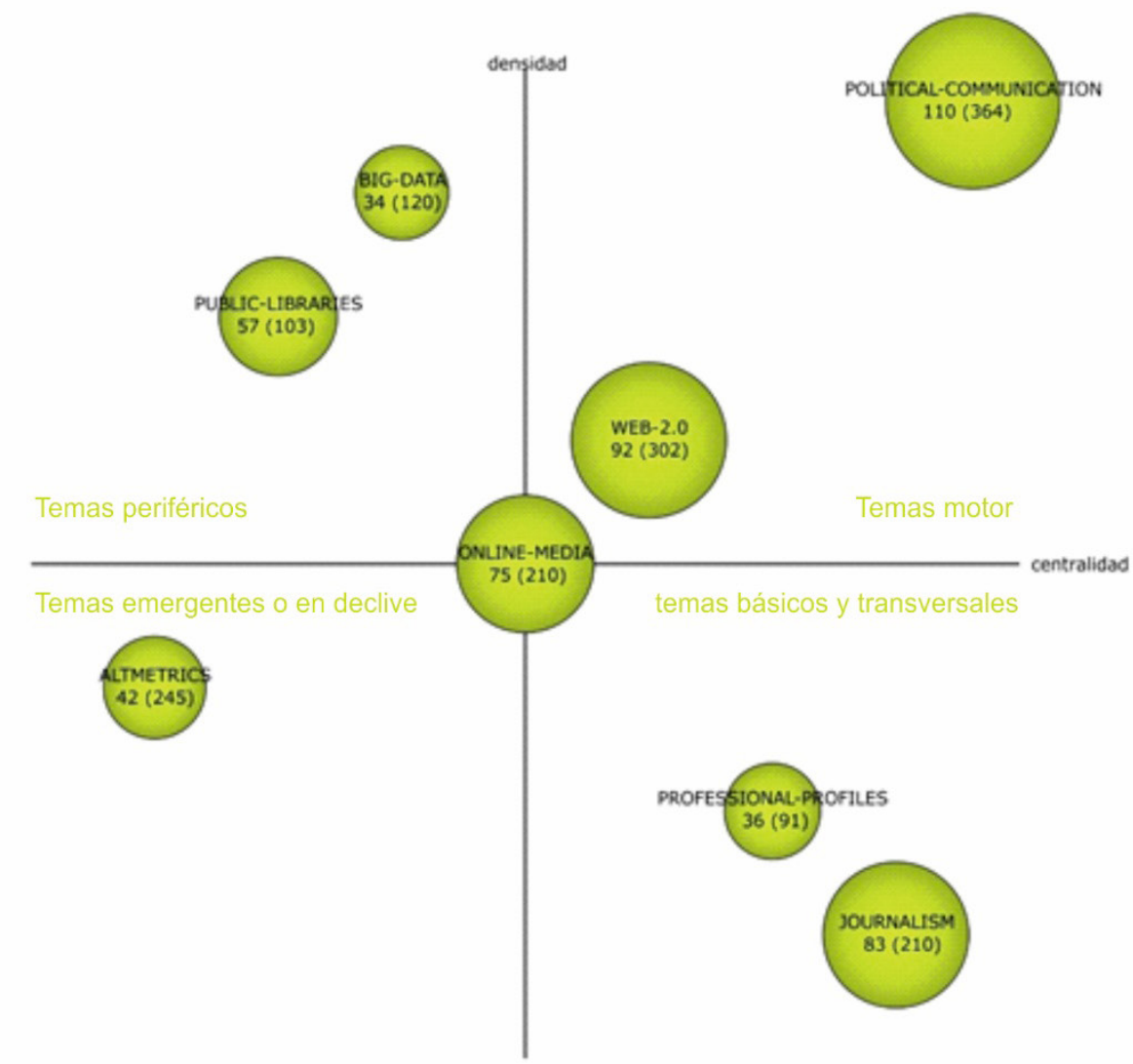

\begin{tabular}{lcccc}
\hline Tema & Cuadrante & Publicaciones & Citas (promedio de citas) & Índice-h \\
\hline POLITICAL-COMMUNICATION & 1 & 110 & $364(3,31)$ & 9 \\
ONLINE-MEDIA & 1 & 75 & $210(2,80)$ & 8 \\
WEB-2.0 & 1 & 92 & $302(3,28)$ & 9 \\
JOURNALISM & 4 & 83 & $210(2,53)$ & 7 \\
PUBLIC-LIBRARIES & 2 & 57 & $103(1,81)$ & 6 \\
BIG-DATA & 2 & 34 & $120(3,53)$ & 6 \\
ALTMETRICS & 3 & 42 & $245(5,83)$ & 8 \\
PROFESSIONAL-PROFILES & 4 & 36 & $91(2,53)$ & 5
\end{tabular}

Figura 3. Mapa estratégico y rendimiento de los temas principales de la revista EPI de 2014 a 2018. Elaboración propia.

Tomando en cuenta la figura anterior, a continuación se presenta el análisis de las principales temáticas desarrolladas por la comunidad en El profesional de la información, considerando su productividad, citas y posición dentro de la estructura intelectual, así como los temas cubiertos por estos.

El tema POLITICAL-COMMUNICATION es el más productivo en el período analizado con 110 publicaciones y 364 citas. Este tema se encuentra incluido dentro de los Temas motor (C1) y cubre principalmente temas relacionados con el uso de redes sociales, análisis de campañas electorales y la relación entre ambos temas y la comunicación (ver figura 4 (a)).

El segundo tema más productivo es WEB-2.0 con 92 publicaciones y 302 citas, y se encuentra incluido dentro de los Temas motor (C1). Dentro de los temas cubiertos por esté, se encuentran temáticas diversas como gestión de bibliotecas, comunicación corporativa, datos abiertos y visualización de información, que coinciden en el uso de la Web 2.0 (ver figura 4 (c)).

JOURNALISM aparece como un Tema básico o transversal (C4) y es el tercer tema más productivo con 83 publicaciones y 210 citas. Dentro de los temas cubiertos, destacan los medios digitales, la prensa digital, las fuentes de información y el diseño de la propia información (ver figura 4 (d)).

ONLINE-MEDIA se encuentra incluido dentro de los Temas motor (C1) y ocupa la cuarta posición en términos de productividad con 75 publicaciones, pero cuenta con un número de citas similar al tercer tema más productivo. Los temas incluidos dentro de esté, son principalmente aquellos relacionados con periódicos, periodismo en más de una modalidad, incluyendo en línea y diversos aspectos asociados a los medios 


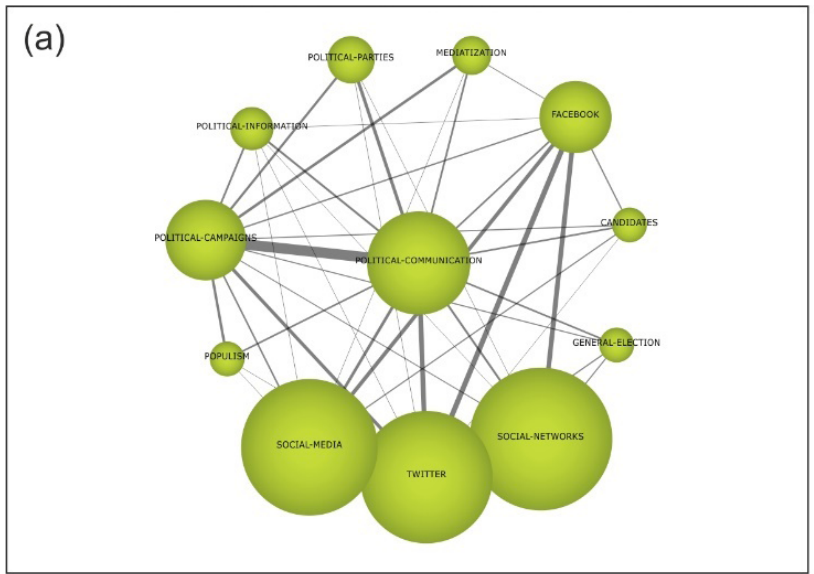

(b)
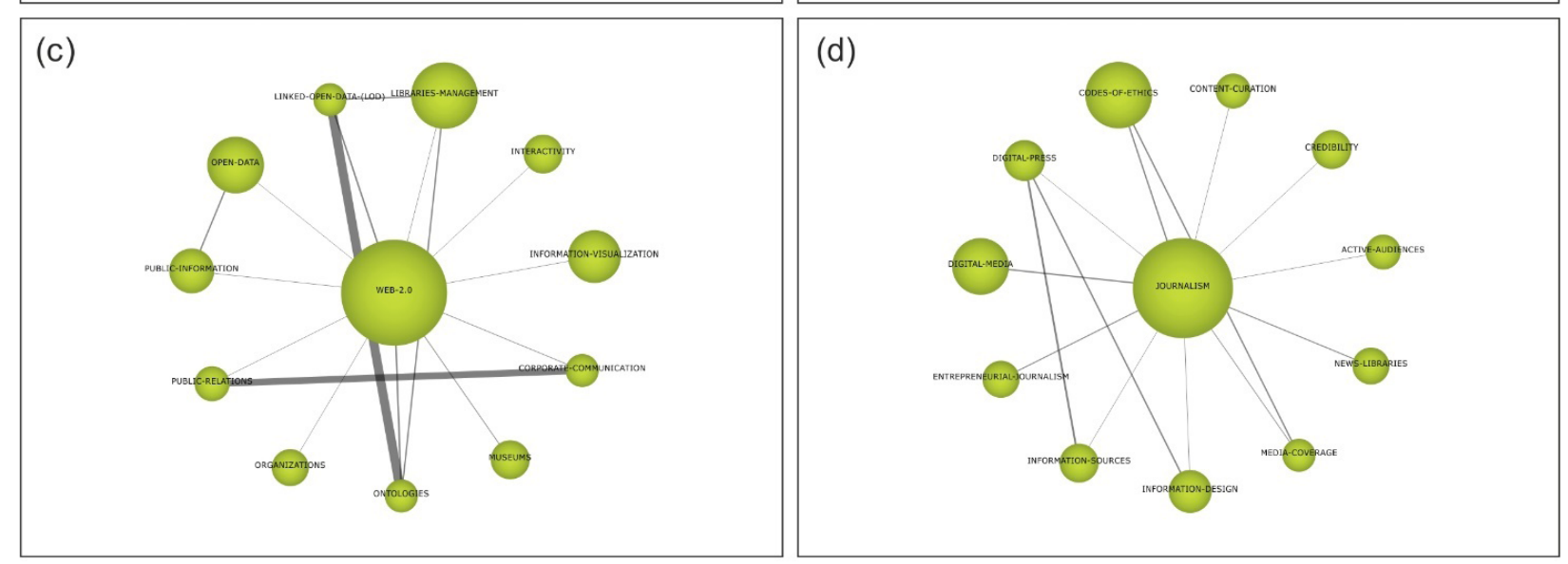

(e)

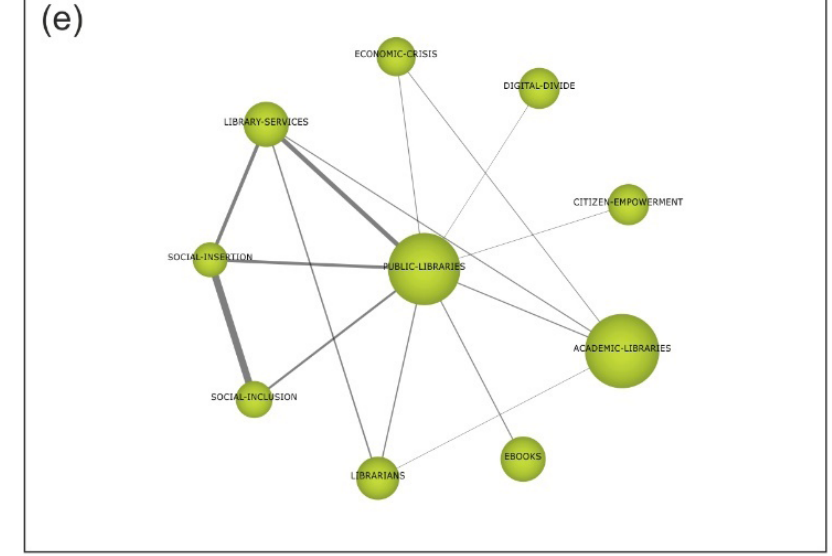

(f)
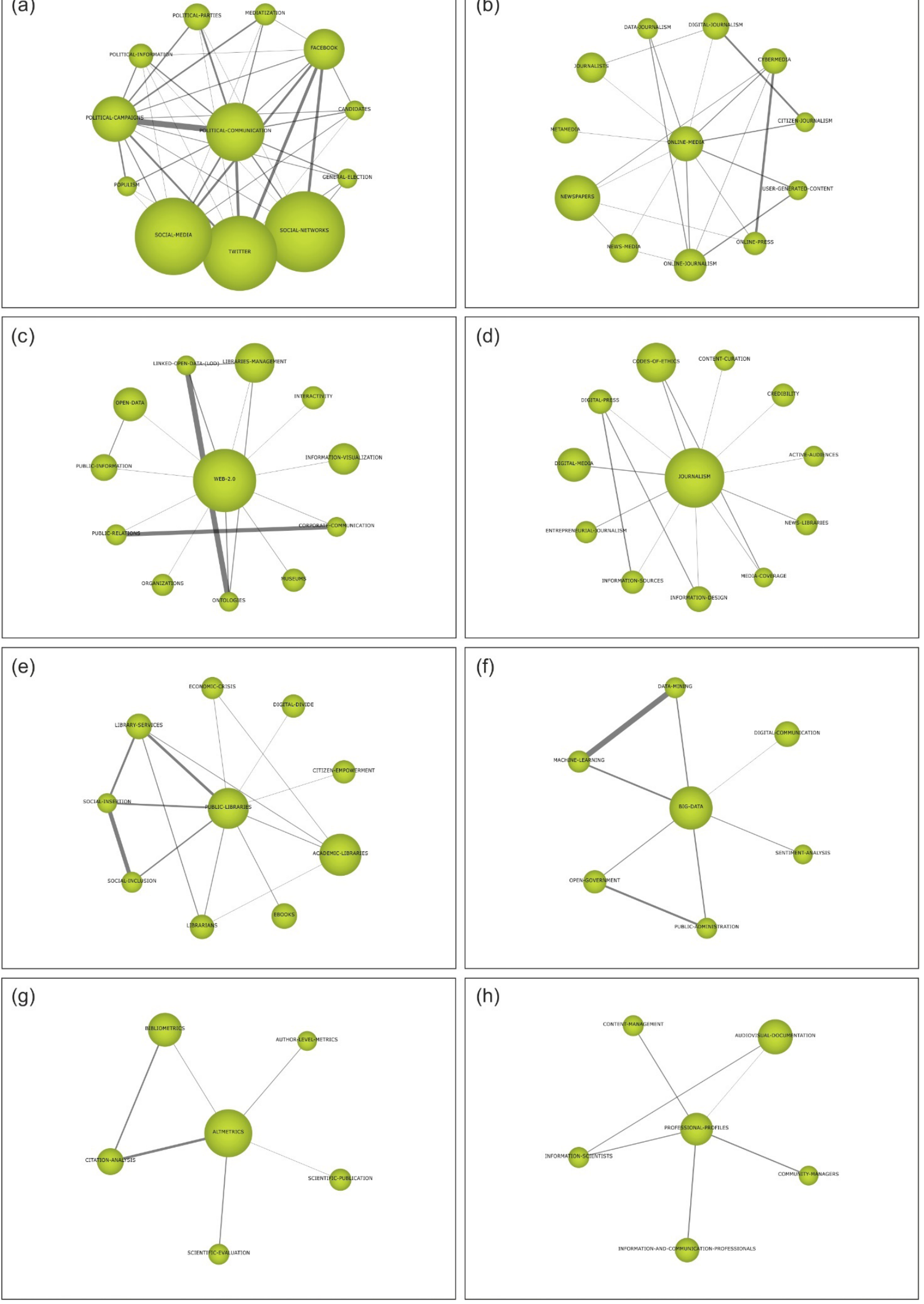

Figura 4. Redes temáticas (a) POLITICAL-COMMUNICATION, (b) ONLINE-MEDIA, (c) WEB-2.0, (d) JOURNALISM, (e) PUBLIC-LIBRARIES, (f) BIG-DATA, (g) ALTIMETRICS y (h) PROFESSIONAL-PROFILES. Elaboración propia. 
(ver figura 4 (b)).

En la quinta posición se encuentra PUBLIC-LIBRARIES con 57 publicaciones y 103 citas. Este tema se encuentra incluido dentro de los Temas periféricos (C2) y cubre principalmente temas relacionados con las bibliotecas académicas, los servicios de bibliotecas y su relación con la sociedad y con los profesionales de este ámbito (ver figura 4 (e)).

ALTMETRICS, localizado como Tema emergente o en declive (C3), se posiciona como el sexto tema más productivo con 42 publicaciones, pero como uno de los más citados, ya que cuenta con 245 citas. Este tema concentra la investigación en temas bibliométricos, análisis de citas y evaluación científica (ver figura 4 (g))

PROFESSIONAL-PROFILES ocupa la séptima posición en términos productivos con 36 publicaciones dentro del periodo de análisis definido y 91 citas, y se clasifica como un Tema básico o transversal (C4). Dentro de los principales temas cubiertos por este se encuentran los profesionales de la información y la comunicación, así como nuevos perfiles relacionados con la curación de contenidos y la gestión de comunidades (ver figura 4 (h)).

Por último, BIG-DATA, incluido en los Temas periféricos (C2), ocupa la última posición con 34 publicaciones y 120 citas, aspecto que refleja un interés por la comunidad, ya que sus citas se encuentran por encima de las obtenidas por otros temas. Cubre aspectos como machine learning, la explotación de datos y su utilización en servicios de comunicación y administración (ver figura 4 (f)).

\section{Conclusiones}

El tamaño de la literatura relacionada con El Profesional de la Información mostró un notable aumento en el último lustro (2014-2018). Dado el gran volumen de publicaciones y citas recibidas en este campo, se espera que el uso de las mismas se considere parte de otros campos del conocimiento.

La revista EPI ha registrado durante el periodo de 2014 a 2018 un total de 476 publicaciones. Este grupo de publicaciones han registrado hasta el 10 de mayo de 2019 un total de 1.105 citas (882 sin citas propias) en un total de 844 publicaciones, promediando 2,53 citas por cada elemento según la Web of Science. Además, presenta un índice h (h-index) de 13 publicaciones, las cuales concentran un total de 284 citas (281 sin citas propias) en un total de 242 artículos, con un promedio de 21,85 citas por elemento.

Estos indicadores reflejan, entre otras cosas, una calidad en las publicaciones al tener un bajo porcentaje de autocitas y un elevado número de publicaciones donde estas se citan. Además, las publicaciones incluidas dentro del índice-h (h-index), consideradas como las publicaciones más relevantes dentro del periodo evaluado presentan un promedio de citas superior a las 21 repeticiones en un periodo medio de 5 años, lo que demuestra una actualidad de las mismas y un interés por parte de la comunidad en estas.

Por otro lado, los principales temas utilizados en El profesional de la información son: POLITICAL-COMMUNICATION, WEB-2.0, JOURNALISM, ONLINE-MEDIA, PUBLIC-LIBRARIES, ALTMETRICS, PROFESSIONAL-PROFILES y BIG-DATA. Dentro de estos temas, POLITICAL-COMMUNICATION, WEB-2.0 y JOURNALISM han sido los más productivos, con 110, 92 y 83 publicaciones, respectivamente.

En términos de estructura intelectual, los temas más relevantes para la confección del núcleo de un área de conocimiento, concepto o agente, son los Temas motor (C1) y Temas básicos o transversales (C2), que en este caso son POLITICAL-COMMUNICATION, ONLINE-MEDIA, WEB-2.0, JOURNALISM y PROFESSIONAL-PROFILES. En este sentido, es posible ver que los tres temas más productivos se encuentran dentro del cuadrante que conforman el núcleo de la revista, lo que confirma su relevancia en el desarrollo de las líneas de investigación.

Por otra parte, en relación con el análisis de la revista entre los años 2006 y 2017 de López-Robles, Guallar, et al. (2019) , se aprecia que en los años recientes, 2014-2018, existe una mayor presencia de las temáticas relacionadas con la Comunicación. Esta tendencia se aprecia con claridad si observamos los temas que se han señalado antes como los más utilizados y los más productivos.

Asimismo, y en relación con el artículo mencionado, en la tabla 2 se muestra la columna 2014-2018 con los resultados de la presente investigación junto a las columnas correspondientes a 2014, 2015, 2016 y 2017 del estudio de 12 años de EPI de López-Robles, Guallar et al 2019 (el año más reciente, 2018, no fue analizado en el citado estudio).

\begin{tabular}{|c|c|c|c|c|c|}
\hline Tema & $\begin{array}{c}2014- \\
2018\end{array}$ & 2014 & 2015 & 2016 & 2017 \\
\hline $\begin{array}{l}\text { POLITICAL-COMMU- } \\
\text { NICATION }\end{array}$ & C1 & & & & \\
\hline WEB-2.0 & C1 & & & C3 & \\
\hline JOURNALISM & C4 & & C4 & C4 & C4 \\
\hline ONLINE-MEDIA & C1 & & $C 2$ & C4 & \\
\hline PUBLIC-LIBRARIES & $C 2$ & & & & \\
\hline ALTMETRICS & C3 & & & & \\
\hline $\begin{array}{l}\text { PROFESSIONAL-PRO- } \\
\text { FILES }\end{array}$ & C4 & C1 & & & \\
\hline BIG-DATA & $C 2$ & $C 2$ & & C4 & C4 \\
\hline
\end{tabular}

Tabla 2. Posicionamiento de los temas principales con respecto a la investigación de López-Robles, Guallar, et al. (2019). 
De las temáticas identificadas, WEB-2.0 en el presente análisis se localiza en el cuadrante C1, mientras que en el análisis anual (menos 2018) se encuentra en el C3 en el año 2016. Por su parte, JOURNALISM ocupa el cuadrante C4 durante 2014-2018 y la misma posición los años 2015, 2016 y 2017. PROFESSIONAL-PROFILES aparece actualmente como C4 y C1 en 2014, así como BIG-DATA en el cuadrante C2, mientras que en el análisis anual ocupa los cuadrantes C2 (2014) y C4 (2016 y 2017). Finalmente, POLITICAL-COMMUNICATION, PUBLIC-LIBRARIES y ALTMETRICS son las únicas embebidas en otras temáticas en la investigación de López-Robles, Guallar, et al. (2019). Sin embargo, esto no contraviene a lo observado en la presente investigación, ya que las temáticas que cubren a estas se encuentran en posiciones similares a las que ocupan en el análisis de 2014 a 2018.

En este sentido, es importante mencionar que el análisis realizado se basa en el análisis de las palabras claves incluidas en los artículos y su co-ocurrencia, situación que se puede ver afectada por la orientación que dan los autores a las palabras claves vinculadas a cada publicación. En lo que respecta a la revista EPI se detecta que existe un control y seguimiento en este tipo de acciones, por lo que los mapas finales reflejan de manera coherente las líneas desarrolladas durante el periodo evaluado.

Finalmente, como posibles líneas futuras de investigación se plantea la evaluación de otras revistas del área durante el mismo periodo de tiempo, con el objetivo de analizar de qué forman interactúan con el desarrollo del área de conocimiento y con El profesional de la información. Además, se plantea un análisis en mayor profundidad en términos de productividad y relación entre autores y organizaciones, identificando los principales grupos de investigación por región o temáticas, de cara a la identificación de tendencias y oportunidades.

\section{Agradecimientos}

Este artículo forma parte del trabajo del grupo de investigación consolidado Cultura i Continguts Digitals, ref. 2017SGR422. Asimismo, los autores J. R. López-Robles y N. K. Gamboa-Rosales agradecen el apoyo del CONACYT-Consejo Nacional de Ciencia y Tecnología (México) y la DGRI-Dirección General de Relaciones Exteriores (México) para la realización de este estudio. Además, este trabajo ha sido apoyado por el Fondo Europeo de Desarrollo Regional con la subvención TIN2016-75850-R.

\section{Referencias}

Abadal, E. (2017). Revistas científicas. Situación actual y retos de futuro. Barcelona: Edicions Universitat Barcelona.

Abadal, E. (2018). ¿Cómo han cambiado BiD y las revistas españolas de documentación en los últimos veinte años? BiD: textos universitaris de biblioteconomia i documentació, (40), 1-10. https://dx.doi. org/10.1344/BiD2018.40.11
Arquero Avilés, R., y Río Sadornil, J. L. (2002). Trayectoria y estudio de producción de la Revista «Documentación de las Ciencias de la Información» en su XXV Aniversario (1976-2001). Documentación de las Ciencias de la Información, (25), 95-114. Recuperado de https://core. ac.uk/download/pdf/38813645.pdf

Calderon Garrido, D., y Carnicer, J. G. (2018). Anàlisi bibliomètrica de la producció científica sobre educació musical en el període 2007-2016 en revistes incloses en JCR. BiD: textos universitaris de biblioteconomia i documentació, (41). https://dx.doi.org/10.1344/BiD2018.41.9

Callon, M., Courtial, J. P., y Laville, F. (1991). Co-word analysis as a tool for describing the network of interactions between basic and technological research: The case of polymer chemsitry. Scientometrics, 22(1), 155-205. https://doi.org/10.1007/BF02019280

Callon, M., Courtial, J. P., y Penan, H. (1995). Cienciometría: La medición de la actividad científica: de la bibliometría a la vigilancia tecnológica. Gijón: Trea.

Castillo-Esparcia, A., Rubio-Moraga, A. L., y Almansa-Martínez, A. (2012). La investigación en Comunicación. Análisis bibliométrico de las revistas de mayor impacto del ISI. Revista Latina de Comunicación Social, 11. https://doi.org/10.4185/RLCS-067-955-248-270

Cobo, M. J. (2012). SciMat: herramienta software para el análisis de la evolución del conocimiento científico. Propuesta de una metodología de evaluación. Granada: Universidad de Granada.

Cobo, M. J., López-Herrera, A. G., Herrera-Viedma, E., y Herrera, F. (2011). An approach for detecting, quantifying, and visualizing the evolution of a research field: A practical application to the Fuzzy Sets Theory field. Journal of Informetrics, 5(1), 146-166. https://doi. org/10.1016/j.joi.2010.10.002

Cobo, M. J., López-Herrera, A. G., Herrera-Viedma, E., y Herrera, F. (2012). SciMAT: A new science mapping analysis software tool. Journal of the American Society for Information Science and Technology, 63(8), 1609-1630. https://doi.org/10.1002/asi.22688

Delgado-López-Cózar, E. (2001). Las revistas españolas de ciencias de la documentación: productos manifiestamente mejorables. El profesional de la información, 10(12), 46-56. Recuperado de http:// hdl.handle.net/10760/34267

Ferran-Ferrer, N., Guallar, J., Abadal, E., y Server, A. (2017). Research methods and techniques in Spanish library and information science journals (2012-2014). Information Research, 22(1). Recuperado de http://hdl.handle.net/10760/32191

Gonzalez-Alcaide, G., Gorraiz, J., y Hervas-Oliver, J. L. (2018). On the use of bibliometric indicators for the analysis of emerging topics and their evolution: spin-offs as a case study. El profesional de la información, 27(3), 493-510. https://doi.org/10.3145/epi.2018.may.04

Goyanes, M., Rodríguez-Gómez, E. F., y Rosique-Cedillo, G. (2018). Investigación en comunicación en revistas científicas en España (2005-2015): de disquisiciones teóricas a investigación basada en evidencias. El profesional de la información, 27(5), 1281-1291. https:// doi.org/10.3145/epi.2018.nov.11

Guallar, J., Ferran Ferrer, N., Abadal, E., y Server, A. (2017). Revistas científicas españolas de información y documentación: análisis temático y metodológico. El profesional de la información, 26(5), 947-960. https://doi.org/10.3145/epi.2017.sep.16

He, Q. (1999). Knowledge discovery through co-word analysis. Library Trends, 48(1), 26. Recuprado de http://hdl.handle.net/2142/8267

López-Ornelas, M., y Cordero-Arroyo, G. (2005). Un intento por definir las características generales de las revistas académicas electró- 
nicas. Revista Razón y Palabra, 10(43), 1-33.

López-Robles, J. R., Cobo, M. J., Guallar, J., Otegi-Olaso, J. R., y Gamboa-Rosales, N. K. (2019). Análisis temático y conceptual de revistas científicas mediante SciMAT: el caso de El Profesional de la Información (EPI), 2013-2018. CRECS2019, 9a Conferencia internacional sobre revistas de Ciencias Sociales y Humanidades, Universidad de la Rioja, Logroño, 23-24 mayo 2019 Recuperado de http:// hdl.handle.net/10760/38374

López-Robles, J. R., Guallar, J., Otegi-Olaso, J. R., y Gamboa-Rosales, N. K. (2019). El profesional de la información (EPI): bibliometric and thematic analysis (2006-2017). El profesional de la información, 28(4), e280417. https://doi.org/10.3145/epi.2019.jul.17

López-Robles, J. R., Otegi-Olaso, J., R., Porto-Gómez, I., Gamboa-Rosales, H., y Gamboa-Rosales, N. K. (2019). Understanding the intellectual structure and evolution of Competitive Intelligence: a bibliometric analysis from 1984 to 2017. Technology Analysis \& Strategic Management, 1-16. https://doi.org/10.1080/09537325.2019.1686136

López-Robles, J. R., Otegi-Olaso, J. R., Arcos, R., Gamboa-Rosales, N. K., y Gamboa-Rosales, H. (2018). Mapping the structure and evolution of JISIB: A bibliometric analysis of articles published in the Journal of Intelligence Studies in Business between 2011 and 2017. Journal of Intelligence Studies in Business, 8(3). Recuperado de https://ojs. hh.se/index.php/JISIB/article/view/325

López-Robles, J. R., Otegi-Olaso, J. R., Gamboa-Rosales, N. K., Gamboa-Rosales, H., y Cobo, M. J. (2018). 60 Years of Business Intelligence: A Bibliometric Review from 1958 to 2017. New Trends in Intelligent Software Methodologies, Tools and Techniques: Proceedings of the 17th International Conference SoMeT_18. https://doi. org/10.3233/978-1-61499-900-3-395

López-Robles, J. R., Otegi-Olaso, J. R., Porto-Gómez, I., y Cobo, M. J. (2019). 30 years of intelligence models in management and business: A bibliometric review. International Journal of Information Management, (48), 22-38. https://doi.org/10.1016/j.ijinfomgt.2019.01.013

López-Robles, J. R., Otegi-Olaso, J. R., Porto-Gómez, I., Gamboa-Rosales, N. K., Gamboa-Rosales, H., y Robles-Berumen, H. (2018). Bibliometric Network Analysis to Identify the Intellectual Structure and Evolution of the Big Data Research Field. International Conference on Intelligent Data Engineering and Automated Learning. https://doi. org/10.1007/978-3-030-03496-2_13

López-Robles, J. R., Otegi-Olaso, J. R., y Porto Gómez, I. (2018). Bibliometric analysis of worldwide scientific literature in Project Management Techniques and Tools over the past 50 years: 1967-2017. Research and Education in Project Management (Bilbao, 2018), 49.

López López, P., Díaz Hernández, R. A., Angulo Benítez, S., y Giménez Toledo, E. (2001). Estudio bibliométrico y de calidad de la revista general de información y documentación (1991-2000). Revista General de Información y Documentación, 11(1), 11-32. Recuperado de http://hdl.handle.net/10760/8627

Pérez Álvarez-Ossorio, J. R. (1997). Cobertura temática y procedencia institucional de los artículos publicados en la Revista Española de Documentación Científica en sus veinte años de existencia. Revista Espanola de Documentacion Cientifica, 20(3), 290-298. https://doi. org/10.3989/redc.1997.v20.i3.584

Powell, T. H., Kouropalatis, Y., Morgan, R. E., y Karhu, P. (2016). Mapping knowledge and innovation research themes: Using bibliometrics for classification, evolution, proliferation and determinism. International Journal of Entrepreneurship and Innovation Management, 20(3-4), 174-199. https://doi.org/10.1504/IJEIM.2016.077960
Somoza, M., Guallar, J., Rodríguez-Gairín, J. M., y Abadal, E. (2017). Presencia de revistas españolas en bases de datos internacionales. En Abadal, E. (ed.) Revistas científicas: situación actual y retos de futuro (pp. 161-178). Barcelona: Universitat de Barcelona.

Sternitzke, C., y Bergmann, I. (2009). Similarity measures for document mapping: A comparative study on the level of an individual scientist. Scientometrics, 78(1), 113-130. https://doi.org/10.1007/ s11192-007-1961-z

\section{CV}

José Ricardo López-Robles. Ingeniero Industrial y de Sistemas por el Instituto Tecnológico y de Estudios Superiores de Monterrey (México, 2007), Master en Dirección de Proyectos por la Universidad del País Vasco/Euskal Herriko Unibertsitatea (España, 2010) y Master en Dirección de Empresas por la Escuela de Negocios Europea de Barcelona (España, 2017). Ha sido galardonado con el Premio Iberoamericano "Veta de Plata 2016" en la categoría "Ciencia y Tecnología" y actualmente es candidato a doctor por la Universidad del País Vasco/Euskal Herriko Unibertsitatea (España). https://twitter.com/ rlopezrobles

Javier Guallar. Doctor en Información y Documentación por la Universidad de Barcelona y profesor del Departamento de Biblioteconomía, Documentación y Comunicación Audiovisual de esta universidad. Es director del posgrado Contenidos Social Media, miembro del Grupo de investigación "Cultura y Contenidos digitales" y del Centro de investigación en Información, Comunicación y Cultura CRICC, todos ellos de la Universidad de Barcelona. Es subdirector de la revista El profesional de la información, y director de las colecciones de libros El profesional de la información y EPI Scholar en Editorial UOC. https:// twitter.com/jguallar

Nadia Karina Gamboa-Rosales. Ingeniera Química por la Universidad Autónoma de Zacatecas. Es licenciada y doctora en Ingeniería Química por la Universidad del País Vasco. Ha participado activamente en proyectos de cooperación, innovación, energía y fabricación avanzada. Actualmente está cursando una Maestría en Administración de Empresas en ENEB Business School y colaborando como investigadora en la Unidad Académica de Ingeniería Eléctrica de la Universidad Autónoma de Zacatecas (México). https://twitter.com/karinagamboa

Jose Ramón Otegi-Olaso. Ingeniero con MBA y doctor en Gestión de Proyectos, especializado en gestión de la innovación. Es profesor de la Universidad del País Vasco/Euskal Herriko Unibertsitatea en Bilbao (España), donde coordina los programas de máster y doctorado en Dirección de Proyectos. Ha trabajado como experto externo en tareas de asistencia técnica para gobiernos locales, regionales y de la UE. https:// twitter.com/joserraotegi 
Manuel Jesús Cobo. Ingeniero en Informática por la Universidad de Jaén (2007), y Doctor en Ciencias de la Computación por la Universidad de Granada (2011). Actualmente es profesor titular de universidad en el Departamento de Ingeniería Informática de la Universidad de Cádiz. Sus principales líneas de investigación se centran en la bibliometría, el análisis de mapas científicos, así como las redes bibliométricas y su visualización. Además, mantiene otras líneas de investigación relacionadas con la toma de decisiones en grupo, minería de datos, minería de textos, así como telemedicina. Cuenta con más de 60 publicaciones, entre artículos y conferencias, y es el desarrollador de SciMAT. https://twitter.com/mjcobomartin

\section{.}

\section{Observatorio de Cibermedios}

https://observatoriocibermedios.upf.edu/

\begin{tabular}{l|ll} 
upf. & $\begin{array}{l}\text { Universitat } \\
\text { Pompeu Fabra } \\
\text { Barcelona }\end{array}$ & $\begin{array}{l}\text { Departamento } \\
\text { de Comunicación } \\
\text { Grupo DigiDoc }\end{array}$
\end{tabular} Barcelona

Grupo DigiDoc

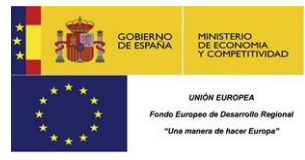

El Observatorio de Cibermedios es una producción del Grupo de Investigación en Documentación Digital y Comunicación Interactiva (DigiDoc) del Departamento de Comunicación de la Universitat Pompeu Fabra.
El Observatorio de Cibermedios (OCM) forma parte del proyecto del Plan Nacional "Creación y contenido interactivo en la comunicación de información audiovisual: audiencias, diseño, sistemas y formatos". CSO2015-64955-C4-2-R (MINECO/ FEDER), Ministerio de Economía y Competitividad (España).

INFORME DIGIDOC - EPI

VISIBILIDAD WEB DE PORTALES DE TELEVISIÓN Y RADIO EN ESPAÑA: ¿OUÉ MEDIOS LLEVAN ACABO UN MEJOR POSICIONAMIENTO EN BUSCADORES? 